\title{
Free-swimming behaviour of Acartia clausi (Copepoda: Calanoida) under turbulent water movement
}

\author{
Enric Saiz*, Miquel Alcaraz \\ Institut de Ciències del Mar, P. Nacional s/n, E-08039 Barcelona, Spain
}

\begin{abstract}
The free-swimming behaviour of Acartia clausi under controlled food and turbulence conditions has been studied in the laboratory. Three experimental conditions were tested: Calm (no turbulence), Low Turbulence ( $\varepsilon$ : horizontal: $1.1 \mathrm{~mm}^{2} \mathrm{~s}^{-3}$; vertical: $0.7 \mathrm{~mm}^{2} \mathrm{~s}^{-3}$ ), and High Turbulence ( $\varepsilon$ : horizontal: $4.9 \mathrm{~mm}^{2} \mathrm{~s}^{-3}$; vertical: $5.4 \mathrm{~mm}^{2} \mathrm{~s}^{-3}$ ). Feeding activity, as estimated by the frequency of feeding bouts, increased in turbulent water motion by a factor of 2.7 . Turbulence also induced an increase in the frequency of and velocity displayed in jumping behaviour (by 1.7 times for frequency of bouts, by about 1.9 for jump velocity). These results confirm previous theoretical and experimental hypotheses about the importance of small-scale turbulence on zooplankton activity and dynamics.
\end{abstract}

\section{INTRODUCTION}

The role of small-scale turbulence in zooplankton activity and dynamics has acquired new interest from recent results obtained from microcosm experiments (Nixon et al. 1979, Oviatt 1981, Alcaraz et al. 1988 , 1989, Saiz \& Alcaraz 1991), theoretical models (Rothschild \& Osborn 1988, Evans 1989, Granata \& Dickey 1991 ) and field evidence (Kiørboe et al. 1990, Sundby \& Fossum 1990). Nevertheless, although some trends can be outlined (an increase in encounter rates and changes in activity and production), evidence of the effects of small-scale turbulence on zooplankton is frequently indirect and our knowledge is still scarce.

In the last decade the use of high-speed cinematography (Alcaraz et al. 1980, Koehl \& Strickler 1981) and video-recording (Strickler 1985) has shown the wide behaviour repertoire of zooplankton and its feeding plasticity, which stresses the importance of studying individual behaviour in order to understand certain aspects of zooplankton activity besides using the classical black box approach (incubations). This approach was used in the recent studies of Costello

\footnotetext{
- Present address: Skidaway Institute of Oceanography, PO Box 13687, Savannah, Georgia 31416. USA
}

et al. (1990) and Marrasé et al. (1990) to demonstrate changes in the behaviour of copepods under controlled turbulent conditions, which confirmed previous hypotheses derived from theoretical models (Strickler 1985, Rothschild \& Osborn 1988, Evans 1989) and from microcosm experiments (Perez et al. 1977, Oviatt 1981. Alcaraz et al. 1988, 1989). However, these studies were carried out on restrained (tethered) copepods and further studies are still required under more realistic conditions (free-swimming) and with different species of copepods to be able to extend these results to the field.

This paper reports a first attempt to study the behaviour of free-swimming individuals of the calanoid copepod Acartia clausi under controlled food and turbulent conditions in the laboratory. The experiments consisted of video-recordings of free-swimming copepods under non-turbulent (calm) and turbulent conditions, enabling a detailed description of behaviour patterns and their frequency to be made.

\section{METHODS}

Turbulence estimates. Turbulence was generated by a small Netlon grid $(0.5 \mathrm{~cm}$ mesh size) placed vertically 
in an experimental aquarium $(9 \times 9 \times 12 \mathrm{~cm})$ and attached to a vibrating rod (Fig. 1). A suspension of latex beads $(19.1 \mu \mathrm{m}$ equivalent spherical diameter, ESD) was diluted in the experimental aquarium as a tracer of the fluid motion. We defined a 3-dimensional skeleton inside the aquarium with 27 spatial points distributed symmetrically inside the volume (Fig. 1). The video-camera was focused on each of the 27 spatial points and the movement of particles recorded for $2 \mathrm{~min}$. For this analysis we used a Sony CCD-Iris black and white video-camera SSC-M350CE attached to a horizontally mounted stereomicroscope. Under high light intensity this camera allowed us to 'freeze' pictures. Magnification was $30.5 \times$.

For each of the skeleton points, the $x$ and $z$ coordinates of ca 60 particles at 2 successive times (40 ms) were traced on plastic sheets (ca 1620 particles tracked for each high and low turbulent condition), and digitized to determine instantaneous and fluc-

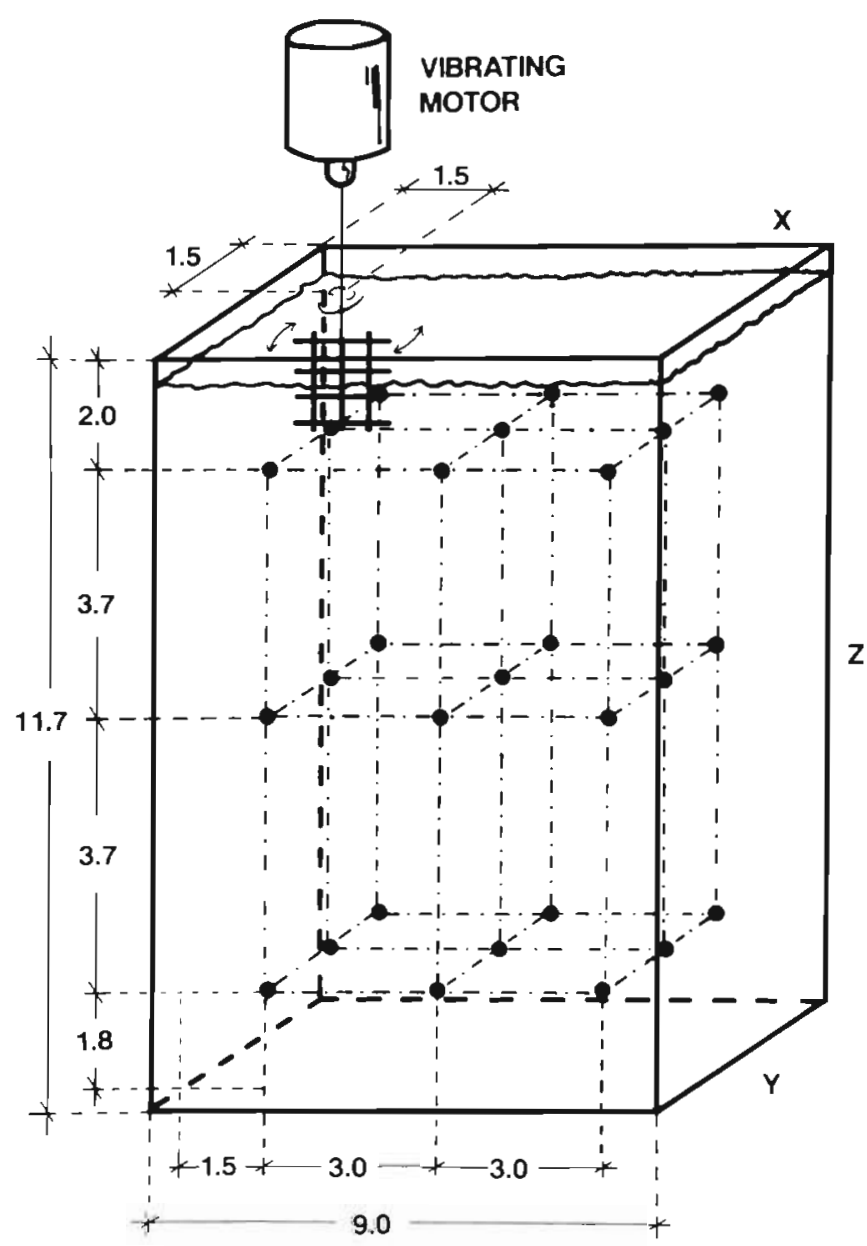

Fig 1 Experimental set-up used for the experiments. The solid circles inside the aquarium represent the 3-D skeleton where particle turbulent motion was recorded. Distances in $\mathrm{cm}$ tuating velocities. Marginal areas of the monitor screen were not considered in order to avoid distortion.

The 3-component turbulent velocity $(Q)$ and the turbulent energy dissipation rate $(\varepsilon)$ for the water motion conditions were estimated following the approach and equations described by Marrasé et al. (1990).

The 3-component turbulent velocity $Q$ (or rootmean-square velocity) was calculated for each point of the skeleton assuming that the dissipation in the $x$ and $y$ directions was similar (isotropic).

Horizontal (considering $x$ and $y$ similar) and vertical turbulent diffusion (HTD and VTD respectively) were also calculated, and the turbulent energy dissipation rates between the nearest skeleton points estimated as

and

$$
\varepsilon_{H}=\mathrm{dHTD} / \mathrm{d} x(\text { or } y)
$$

$$
\varepsilon_{\mathrm{V}}=\mathrm{dVTD} / \mathrm{d} z
$$

This provided us with a rough estimate of the intensity of turbulence in our experiments, and a coarse description of the spatial distribution of the dissipation of turbulent energy in the aquarium.

Experimental procedure. Copepods were captured by means of short oblique net tows $(200 \mu \mathrm{m}$ mesh size) in Barcelona Harbour, Spain, and transported to the laboratory in buckets filled with in situ sea water. Once in the laboratory, copepods were kept in the buckets until Acartia clausi females were sorted and placed in 21 jars filled with in situ water. The jars were fixed on a ferris wheel $(0.2 \mathrm{rpm})$ to keep algae in homogeneous suspension. Every day suspensions of cultures of the algae Thalassiosira weissflogii and Gymnodinium nelsoni were added in excess (until the water became coloured) to ensure an ad libitum food supply. Copepods were kept in these conditions until the experiments started (at least $24 \mathrm{~h}$ ).

Observations were conducted under 2 experimental conditions: non-turbulence (undisturbed or calm) and turbulence ( 2 intensities, high and low). For each experiment, 90 Acartia clausi $q$ were placed in the experimental aquarium filled with $900 \mathrm{ml}$ of a $0.7 \mathrm{~mm}^{3} \mathrm{I}^{-1}$ suspension of Gymnodinium nelsoni. The high density of copepods was required to enhance the probability of individuals appearing in the focused area. Experiments were performed at room temperature 120 $\pm 1^{\circ} \mathrm{C}$ ). After being placed in the experimental vessel, the copepods were allowed to acclimatize to the experimental conditions (food concentration and darkness) for 60 to 90 min before recording started. Filming in all experiments began at approximately local sunset time. After the experiments copepods exhibited normal behaviour and no damage was observed. Videorecording was carried out with a Sony AVC-D5CE CCD video-camera. The observation area was $92 \mathrm{~mm}^{2}$, 
focused in the center of the aquarium. Illumination was provided by a cold light source fitted with a infrared Kodak filter (Ref. 88A). The video-camera used was highly sensitive to infrared light and allowed the experiment to be performed in darkness. We conducted 2 experiments under calm conditions (Calm1 and Calm2), 1 under low turbulence (LowT1) and 2 under high turbulence (HighT1 and HighT2). Only Calm2 and HighT2 were performed consecutively on the same day with the same individuals.

Data analysis. The first 15 to $20 \mathrm{~min}$ of each videotape were analyzed, on a frame-by-frame basis. For experiment Calm2 we extended the period to $55 \mathrm{~min}$ to achieve an analyzed time similar to that of Calm1. This was to compensate for the fact that at the beginning of experiment Calm1 we changed the focus on several occasions to follow the copepods and they therefore appeared in focus more frequently.

The magnification $(14 \times)$ and temporal resolution ( 25 frames $\mathrm{s}^{-1}$, i.e. $40 \mathrm{~ms}$ between frames) allowed us to distinguish 3 different patterns of behaviour: (1) passive behaviour, with no movements; (2) feeding bouts, with movements of the feeding appendages and thoracopods; (3) jumps, escape reactions or fast swimming, about 2 body length displacements in a short period of time (ca 2 to 3 frames).
Only pictures sharply enough in focus to recognize these patterns were used. We realized, however, that in experiments under high turbulent conditions (HighT1 and HighT2) movement was sometimes so fast that feeding bouts not associated to small reorientations of the body (tilts) could have been underestimated in some pictures. To avoid the increased probability of observing passive behaviour under turbulence due to the mechanical advection of individuals, we considered only sequences in which the copepod showed at least one active pattern.

Frequency of feeding bouts and jumps was estimated for the sequences analyzed. By tracking successive copepod positions on plastic sheets frame by frame, velocities of passive sinking, advection (motion of the copepod carried away by the turbulent eddies) and jumps were determined, Jumping velocity under turbulent conditions was corrected to take into account the advection component (velocity vectors for the jumps and their previous advection were drawn on the sheets assuming constant speed and the advection component subtracted). Only sequences in which advection could be determined accurately (i.e. good focus) were used for this analysis. Although passive sinking provided a very small velocity component, velocities for calm conditions were calculated in the same way.
A
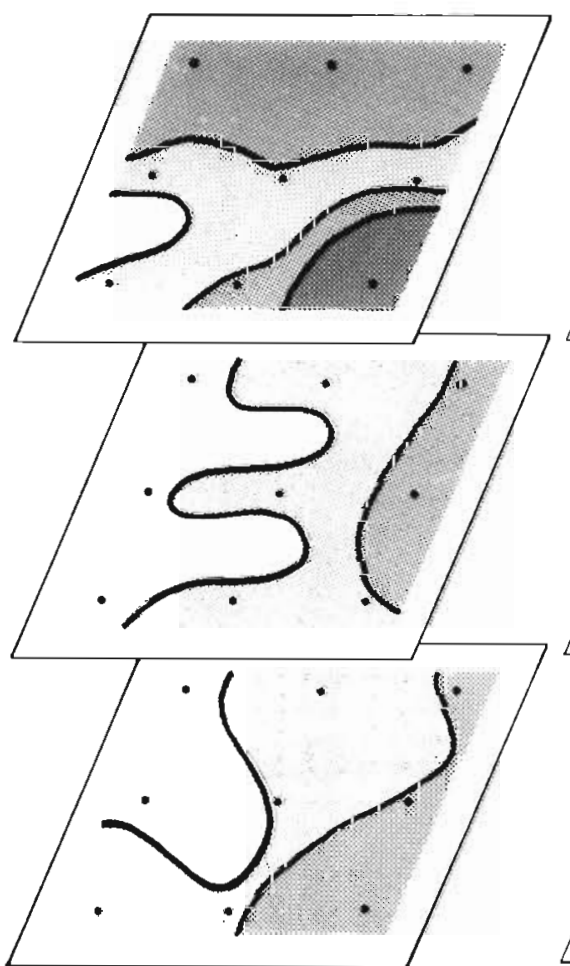

$\square 0.0 .2 \quad \square_{0.2-0.5}$

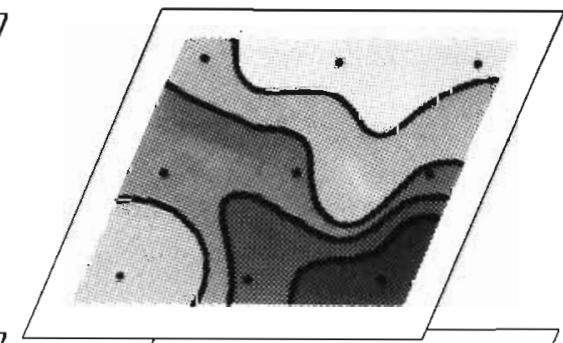

Fig. 2. Spatial distribution of horizontal turbulent energy dissipation rates $\left(\varepsilon_{\mathrm{H}}\right.$ $\mathrm{mm}^{2} \mathrm{~s}^{-3}$ ) in the experimental aquarium under (A) low and (B) high turbulent conditions. The 3 layers where measurements were performed (upper medium and lower) are represented

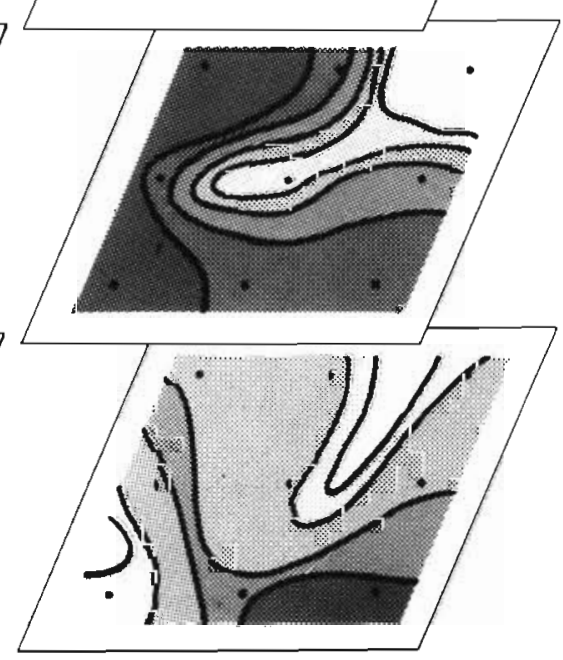

0.5-2 2-8

$>32$ 


\section{RESULTS}

\section{Water motion conditions}

Figs. 2 \& 3 show the spatial distribution in the experimental aquarium of the estimated horizontal and vertical turbulent dissipation rates for the 2 turbulent conditions. We considered that as long as the same forcing input was used throughout the measurements, temporal variability (i.e. that all the skeleton points were not filmed simultaneously) would not be important. That is, even though this picture does not
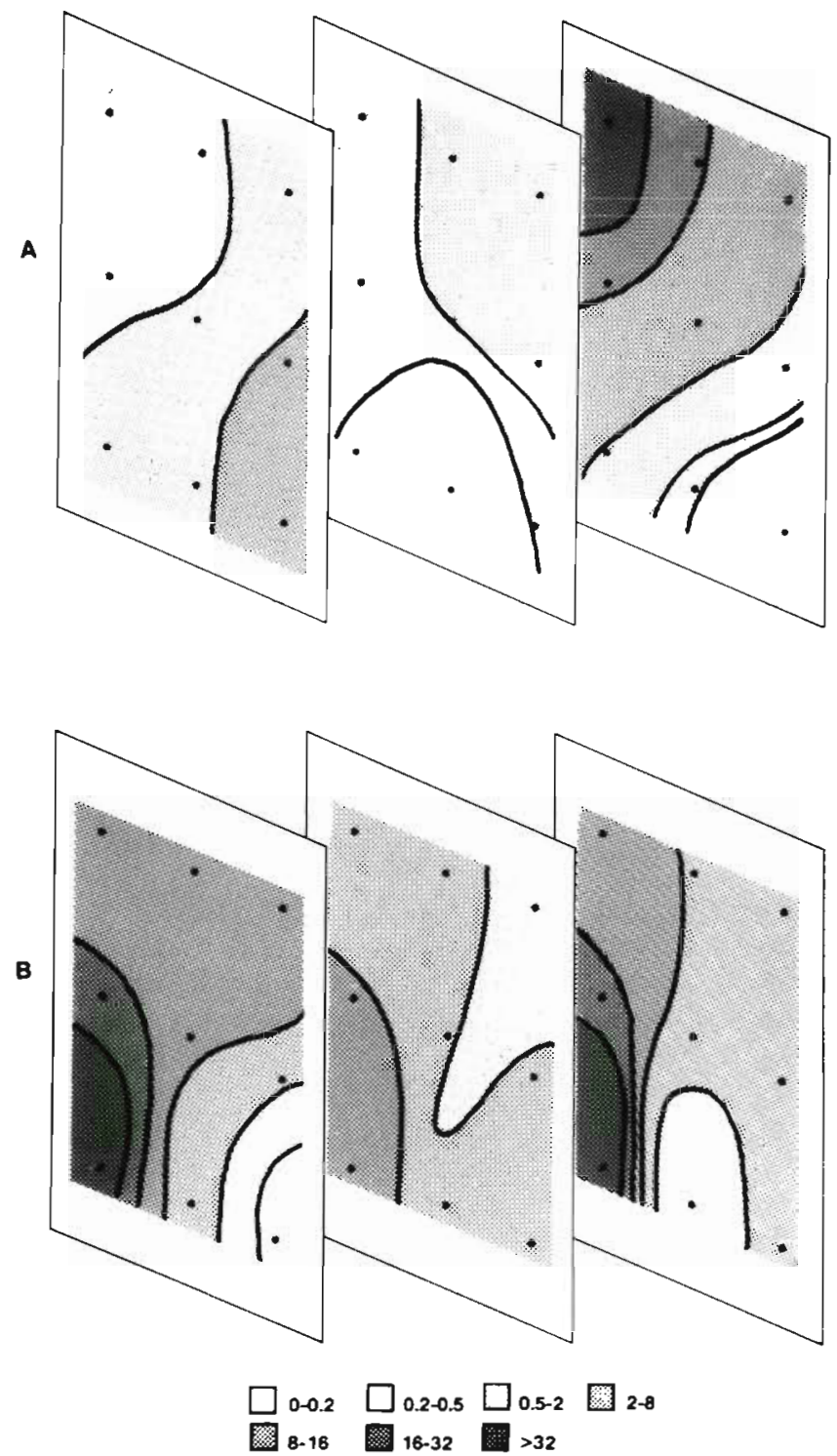

Fig. 3. Spatial distribution of vertical turbulent energy dissipation rates $\left(\varepsilon_{v}, \mathrm{~mm}^{2} \mathrm{~s}^{-3}\right)$ in the experimental aquarium under $(A)$ low and $(B)$ high turbulent conditions. The 3 layers where measurements were performed (front, medium and back) are represented
Table 1. Estimated horizontal and vertical turbulent energy dissipation rates $\left(\varepsilon_{\mathrm{H}}\right.$ and $\varepsilon_{V}$ respectively) for experiments under low and high turbulent conditions. $\varepsilon$ is expressed in $\mathrm{mm}^{2} \mathrm{~s}^{-3}$

\begin{tabular}{|lcccc|}
\hline & \multicolumn{2}{c}{ Low turbulence } & \multicolumn{2}{c|}{ High turbulence } \\
& $\varepsilon_{\mathrm{H}}$ & $\varepsilon_{\mathrm{V}}$ & $\varepsilon_{\mathrm{H}}$ & $\varepsilon_{\mathrm{V}}$ \\
\hline $\mathrm{n}$ & 36 & 18 & 36 & 18 \\
Geom. mean & 1.1 & 0.7 & 4.9 & 5.4 \\
Min. value & 0.06 & 0.06 & 0.003 & 0.4 \\
Max. value & 12.6 & 17.7 & 59.3 & 54.7 \\
& & & &
\end{tabular}

represent true simultaneous measurements, it should give us an idea of the general pattern.

The 3 component turbulent velocity (Q) for low turbulent conditions averaged $4.9 \pm 0.4(\mathrm{SE}) \mathrm{mm} \mathrm{s}^{-1}$, which was significantly lower than the value for high turbulent conditions $\left(11.0 \pm 0.6 \mathrm{~mm} \mathrm{~s}^{-1}\right.$; MannWhitney 2-tailed test, $\mathrm{p}<0.001)$.

Turbulent dissipation rates (Table 1) did not show any significant difference between the horizontal and the vertical components in any of the experimental conditions (Mann-Whitney test). However, vertical and horizontal dissipation rates were higher under high turbulent conditions than under low turbulent conditions (Mann-Whitney 2 -tailed test, $\mathrm{p}<0.001$ ).

\section{Acartia clausi behaviour}

Fig. 4 shows 2 representative drawings of the freeswimming behaviour patterns of Acartia clausi under calm and turbulent conditions. Under calm conditions, A. clausi sank passively through the water and displayed frequent rapid upward bursts of swimming (99 $\pm 4 \mathrm{~ms}$ duration, equal to a distance of $1.5 \pm$ $0.06 \mathrm{~mm}$ ) to maintain its depth position (Figs. $4 \mathrm{~A} \& 5$ ). Feeding bouts were often associated to small tilts and reorientations of the body.

Under turbulent water motion Acartia clausi showed the same patterns of behaviour (passive, jumping and feeding bouts), although there were significant differences in their performance. Thus, instead of sinking, passive behaviour resulted in the copepods being carried away by water currents (advection, Fig. 4B). Velocities for sinking, turbulent advection and jumps (after correction) were determined only for experiments Calm1, LowT1 and HighT1 (Fig. 5). Sinking velocity was on average $1.2 \mathrm{~mm} \mathrm{~s}^{-1}$. Water movement in turbulence experiments increased the velocity of displacement in passive behaviour (advection). Advection velocity under high turbulent conditions was twice that under low turbulent conditions.

Jump velocity under low turbulent conditions (Fig. 5) was significantly higher than under calm conditions (2-tailed Mann-Whitney test, $\mathrm{p}<0.008$ ). Despite the 
A. No turbulence

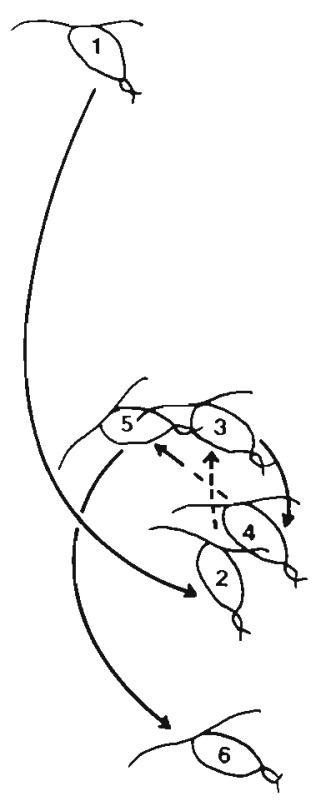

Fig. 4. Acartia clausi. Behaviour under (A) non-turbulent and (B) turbulent conditions. Passive behaviour (continuous line; sinking under calm, advection under turbulence) and jumping (dashed line) patterns are represented

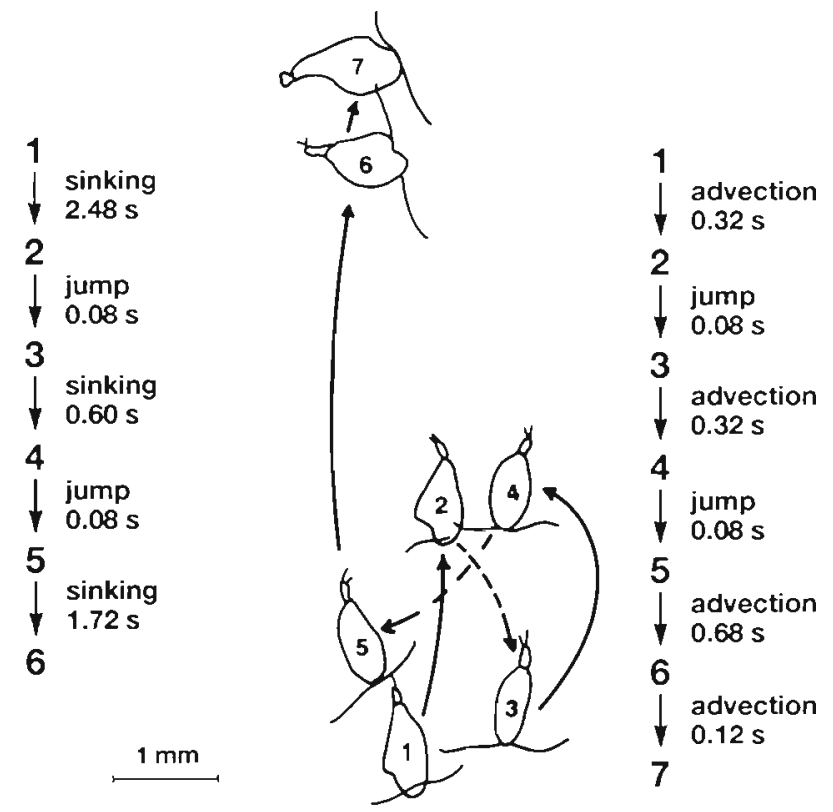

These feeding bouts included 2 patterns of behaviour: one that only involved the movement of feeding appendages and thoracopods with a slow forward motion (hereafter called 'suspension-feeding-like') as well as the same kind of movement of feeding appendages associated to small jumps or tilts of the body, as observed under calm conditions. Mean durations of suspension-feeding bouts were similar under calm and turbulent conditions (Calm: $168 \pm 20 \mathrm{~ms}$; Turbulent: $136 \pm 8 \mathrm{~ms}$ ). We did not quantify any difference in tilt-associated feeding events between conditions. Feeding tilt events usually were shorter than the suspension-feeding ones, for both calm and turbulent conditions, and ranged from 80 to sometimes $280 \mathrm{~ms}$.

\section{DISCUSSION}

\section{Behaviour under calm conditions}

Table 2. Acartia clausi. Frequency of jumps and feeding bouts for the experiments conducted. Total time the copepods were observed is also given

\begin{tabular}{|lrcc|}
\hline Experiment & $\begin{array}{c}\text { Studied } \\
\text { time (s) }\end{array}$ & $\begin{array}{c}\text { Jumps } \\
\mathrm{min}^{-1}\end{array}$ & $\begin{array}{c}\text { Feeding } \\
\text { bouts } \text { min }^{-1}\end{array}$ \\
\hline Calm1 & 95.4 & 41 & 14 \\
Calm2 & 77.3 & 21 & 13 \\
LowT1 & 78.4 & 41 & 39 \\
HighT1 & 47.6 & 59 & 42 \\
HightT2 & 76.7 & 70 & 34 \\
Pooled Calm & 172.7 & 32 & 14 \\
Pooled Turb & 202.7 & 56 & 38 \\
\hline
\end{tabular}

The behaviour exhibited by Acartia clausi under calm conditions in our experiments agreed with previous reports for this and related species (Rosenberg 1980, Paffenhöfer \& Stearns 1988, Jonsson \& Tiselius 1990, Tiselius \& Jonsson 1990). Instead of showing an almost continuous slow swimming pattern (i.e. creation of feeding currents associated with a slow forward motion), A. clausi sank passively through the water and displayed frequent jumps to maintain its depth position (Fig. 4). This behaviour has been related to the search for motile, relatively large prey (Paffenhöfer \& 


\section{SINKING AND FLOW ADVECTION}
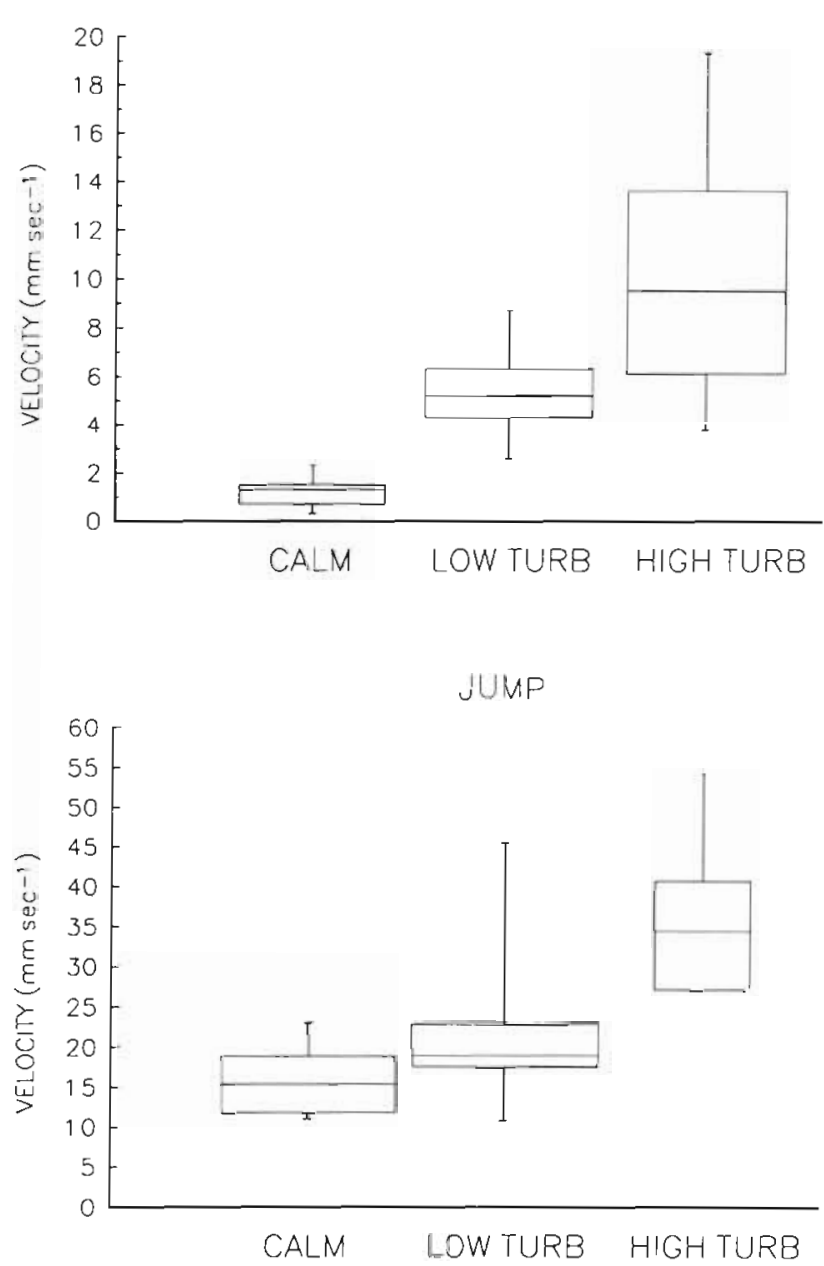

Fig. 5. Acartia clausi. Velocities for sinking, advection and jumping under calm and turbulent conditions. Horizontal line: median; vertical line: range of values; boxes: represent $25 \%$ of values above and below median respectively. The width of each box is proportional to the square root of the size of group it represents

Stearns 1988, Jonsson \& Tiselius 1990). The abundant mechanoreceptors on the first antenna (Barrientos 1980 ) and the structure and action of its mouthparts (Paffenhöfer \& Stearns 1988) also suggest a raptorial behaviour. Passive sinking provokes a low rate of fluid deformation, probably below the critical value for prey avoidance (Haury et al. 1980, Tiselius \& Jonsson 1990). Mean sinking velocities were slightly higher than those previously reported for $A$. clausi $\left(0.3 \mathrm{~mm} \mathrm{~s}^{-1}\right.$, Tiselius \& Jonsson 1990) and A. tonsa (0.8 $\mathrm{mm} \mathrm{s}^{-1}$, Jonsson \& Tiselius 1990). As described by these authors, copepods usually started sinking with a horizontal body orientation but a force (torque) tended to align the copepod along the vertical axis. On several occasions we observed individuals passively sinking head down, and then jumping downward. The frequency of jumps observed was very similar to those reported by the authors mentioned [ $A$. tonsa: ca $60 \mathrm{~min}^{-1}$; A. clausi: (in low light) 54 jumps $\mathrm{min}^{-1}$ ].

Our experiments were conducted with the copepods being offered a moderate algal concentration $\left(0.7 \mathrm{~mm}^{3} \mathrm{l}^{-1}\right)$ and no animal prey present. However, copepods displayed 2 different patterns of feeding activity in our experiments. Suspension-feeding-like behaviour involved short feeding bouts with movements of mouthparts and thoracopods (Rosenberg 1980, Paffenhöfer \& Stearns 1988). This motion generates a ventrally directed flow field (feeding current) used by the copepod to gather food. Due to the magnification used in our experiments $(14 \times)$ this flow field could not be discerned in our observations. The other pattern of behaviour exhibited was raptorial feeding; it was associated to reorientations of the body and short jumps (tilts) and movement of the mouthparts and thoracopods. There is clear evidence that Acartia sp. does not behave as a true suspension-feeder. It spends only little time creating feeding currents (Jonsson \& Tiselius 1990, Tiselius \& Jonsson 1990) compared to 'true' suspension-feeding calanoid copepods (50 to $90 \%$; Paffenhöfer \& Stearns 1988). Acartia can rapidly switch from one feeding behaviour to the other when food conditions change. We conclude that the size (ca $37 \mu \mathrm{m} \mathrm{ESD}$ ) and speed $\left(1.0 \pm 0.05 \mathrm{~mm} \mathrm{~s}^{-1}\right)$ of Gymnodinium nelsoni in our experiments can induce both suspension-like and raptorial feeding patterns. However, under conditions of abundance of smallsized algae (i.e. Thalassiosira weisflogin), Acartia is able to display more a suspension-feeding-like behaviour (pers. obs.).

\section{Behaviour under turbulence}

In our experiments turbulent water motion induced changes in the behaviour of Acartia clausi. Passive behaviour resulted in being carried away instead of sinking (Fig. 4B). The jumping frequency was higher and jumps were faster under turbulence. Both suspension-feeding-like and raptorial behaviour were also displayed with a higher frequency under turbulent conditions

The increase in the frequency of feeding bouts under turbulent conditions is probably a consequence of the enhanced encounter rates between predator and prey under water motion (Rothschild \& Osborn 1988, Costello et al. 1990) and suggests a higher feeding activity. Enhanced activity (i.e. feeding) has been proposed for copepods inhabiting turbulent areas in natural systems (Kiørboe et al. 1990) and in microcosm experiments (Alcaraz et al. 1989). However, higher 
ingestion cannot be inferred from our experiments because capture efficacy could be lower under turbulence. Furthermore, different feeding behaviours (i.e. suspension vs raptorial feeding) could be differently affected. The dependence of mechanoreception for prey capture may make predatory behaviour more sensitive to water turbulence.

The higher frequency and strength of jump in turbulence may be caused by increased mechanical stimulation of antennal mechanoreceptors by small turbulent eddies (Alcaraz et al. 1988, 1989, Costello et al. 1990, Marrasé et al. 1990). It is presently not known which properties of fluid motion are sensed by copepods. Cumulative fluid deformation and/or the rate of deformation seem to be the most important stimuli (Haury et al. 1980, Tiselius \& Jonsson 1990), although pressure pulses have also been proposed (see Gill \& Crisp 1985).

The increase in jumping velocity under turbulent conditions (1.4 times under low turbulent conditions, 2.3 times under high turbulent conditions), coupled with the higher frequency of jumps, may have a considerable effect on the energy budget of the individual. Alcaraz et al. $(1988,1989)$ suggested that the turbulence-induced increase in the frequency of escape reactions probably would result in higher metabolic rates associated to enhanced grazing and accelerated development rates (Alcaraz et al. 1988 , Saiz \& Alcaraz 1991). It is not clear whether this metabolic increase can be significant in the field. The video studies of Costello et al. (1990) and Marrasé et al. (1990) reported an increase in the frequency of escape reactions in tethered Centropages hamatus under turbulent conditions, but a saturation effect appeared after a few minutes. Although we did not consider changes in time in our experiments, we did not observe any attenuation in the response during the first 15 to $20 \mathrm{~min}$ of turbulence. Species-specific differences in the mechanosensorial system and the intensity of turbulence might account for this.

The turbulence generated during our experiments was comparable to the range of turbulent dissipation rates encountered in a variety of natural environments, especially shelf and coastal waters (shelf: 0.1 to $1 \mathrm{~mm}^{2} \mathrm{~s}^{-3}$; coastal: 0.1 to $100 \mathrm{~mm}^{2} \mathrm{~s}^{-3}$; see Marrasé et al. 1990, Granata \& Dickey 1991 and references therein). However, one must be cautious in any direct extrapolation to natural environments. Our stirring mechanism does not generate turbulence in all the ranges an animal can find in the field, and measurements also involved a cut-off in the higher frequencies due to the video resolution and to the size of the area where particles could be tracked. Further, there is an upper limit due to the size of the aquarium (about
$10 \mathrm{~cm}$ ) and a constraint in the scale at which the main input of energy was introduced (related to the size of the grid used). Thus we must consider that our work has focused on a small window of the length scales in the energy spectra that can be found in natural systems. However, the range generated is close to the size of the copepod (about $1 \mathrm{~mm}$ ) and a priori this is the range that should most affect the behaviour of the copepods. Much higher eddies could induce other kind of effects (i.e. transport). Much smaller eddies, in the viscous range, would be dissipated. Although we did not attempt to simulate any particular natural environment, we think that the processes induced by the turbulence we generated in the laboratory can also act in a natural turbulent environment (i.e. all length scales) and be of considerable importance

Our results confirm the influence of small-scale turbulence on copepod activity and behaviour. Although higher activity of zooplankton (feeding, excretion, fecundity) has frequently been reported in relation to hydrodynamic features (Harris \& Male) 1986, Kiørboe \& Johansen 1986, Peterson \& Bellantoni 1987, Kiørboe et al. 1988, 1990), most of the variability observed has been attributed to indirect effects of turbulence on phytoplankton (i.e. changes in food size and quality; see Peterson \& Bellantoni 1987. Kiørboe et al. 1990). Direct effects are difficult to discern in the field. Sundby \& Fossum (1990) observed a close relationship between wind-induced turbulence and feeding of cod larvae (gut contents). Our results, coupled with other experimental evidence mentioned above, indicate that small-scale turbulence can have a relevant effect on activity and behaviour of copepods. Through these changes small-scale turbulence may influence biomass and community structure of zooplankton (Alcaraz et al. 1988, Saiz \& Alcaraz 1991), as well as the control exerted by planktonic herbivores on phytoplankton (Alcaraz et al. 1989). The importance of copepods as a link between producers and higher trophic levels, particularly in pelagic systems prone to human exploitation (Cushing 1989), emphasizes the relevance of these studies. Future field and experimental work may confirm small-scale turbulence as a decisive modulating factor of ecological relationships in pelagic ecosystems.

Acknowledgements. This research was supported by a Postdoctoral Fellowship PF91 46035640 to E.S. and grant CYCIT MAR88-0252 of the Spanish Government. We thank Drs G.-A. Paffenhöfer, P. Tiselius and the anonymous referees for their comments on the manuscript, and Dr C. Marrasé for her helpful advice and comments on turbulence estimation. Mikel Latasa and Marta Porta helped in digitizing tasks, and Antonia Cruz and Maria Velez drew some of the figures. 


\section{LITERATURE CITED}

Alcaraz, M., Paffenhöfer, G. A., Strickler, J. R. (1980) Catching the algae: a first account of visual observations on filter-feeding calanoids. In: Kerfoot, W. C. (ed.) Evolution and ecology of zooplankton communities. Univ. Press of New England, Hanover, p. 241-248

Alcaraz, M., Estrada, M., Marrasé, C. (1989). Interaction between turbulence and zooplankton in laboratary microcosms. In: Klekowski, R. Z., Styczynska-Jurewicz, E., Falkowski, L. (eds.) Proc. 21st Eur. Mar. Biol Symp. Polish Academy of Sciences-Institute of Oceanology. Ossolineum, Gdansk, p. 191-204

Alcaraz, M., Saiz, E., Marrasé, C., Vaqué, D. (1988). Effects of turbulence on the development of phytoplankton biomass and copepod populations in marine microcosms. Mar. Ecol. Prog. Ser. 49:117-125

Barrientos, Y. (1980). Ultrastructure of sensory units of the first antennae of calanoid copepods. M. Sc. thesis, Univ. of Ottawa, Ontario

Costello, J. H., Strickler, J. R., Marrasé, C., Trager, G, Zeller, R., Freise, A. J. (1990). Grazing in a turbulent environment: behavioral response of a calanoid copepod Centropages hamatus. Proc. natn. Acad. Sci. USA 87: 1648-1652

Cushing, D. H. (1989). A difference in structure between ecosystems in strongly stratified waters and in those that are only weakly stratified. J. Plankton Res. 11: 1-13

Evans, G. T. (1989). The encounter speed of moving predator and prey. J. Plankton Res. 11: 415-417

Gill, C. W., Crisp, D. J. (1985). Sensitivity of intact and antennule amputated copepods to water disturbance. Mar. Ecol. Prog. Ser. 21: 221-227

Granata, T C., Dickey, T. D. (1991). The fluid mechanics of copepod feeding in a turbulent flow: a theoretical approach. Progr. Oceanogr. 26: 243-261

Harris, R. P., Malej, A. (1986). Diel patterns of ammonium excretion and grazing rhythms in Calanus helgolandicus in surface stratified waters. Mar. Ecol. Prog. Ser. 31: 75-85

Haury, L. R., Kenyon, D. E. (1980). Experimental evaluation of the avoidance reaction of Calanus finmarchicus. J. Plankton Res. 2: 187-202

Jonsson, P. R. Tiselius, P. (1990). Feeding behaviour prey detection and capture efficiency of the copepod Acartia tonsa feeding on planktonic ciliates. Mar. Ecol. Prog. Ser. 60: $35-44$

Kiørboe, T., Johansen, K. (1986). Studies of a larval herring (Clupea harengus L.) patch in the Buchan area. 4. Zooplankton distribution and productivity in relation to hydrographic structures. Dana 6: 37-51

Kiørboe, T., Kaas, H., Kruse, B., Møhlenberg, F., Tiselius, P., Aertebjerg, G. (1990). The structure of the pelagic food web in relation to water column structure in the Skagerrak. Mar. Ecol. Prog. Ser. 59: 19-32

This article was presented by T. Kiorboe, Charlottenlund, Denmark
Kiørboe, T., Munk, P., Richardson, K., Christensen, V., Paulsen, H. (1988). Plankton dynamies and larval herring growth drift and survival in a frontal area. Mar. Ecol. Prog. Ser. 44: 205-219

Koehl, M. A. R.. Strickler, J. R. (1981). Copepod feeding currents: food capture at low Reynolds number. Limnol. Oceanogr. 26: 1062-1073

Marrasé, C., Costello, J. H., Granata, T, Strickler, J. R. (1990). Grazing in a turbulent environment: energy dissipation encounter rates and efficacy of feeding currents in Centropages hamatus. Proc. natn. Acad. Sci. USA 87: $1653-1657$

Nixon, S. W., Oviatt, C. A., Buckley, B. A. (1979). Turbulent mixing in marine microcosms - some relative measures and ecological consequences. In: Jacoff, J. S. (ed.) Advances in marine environmental research. U.S. E.P.A. 600/9-79-035, Narragansett, p. 382-409

Oviatt, C. A. (1981). Effects of different mixing schedules on phytoplankton zooplankton and nutrients in marine microcosms. Mar. Ecol. Prog. Ser. 4: 57-67

Paffenhöfer, G. A., Stearns, D. E. (1988). Why is Acartia tonsa (Copepoda: Calanoida) restricted to nearshore environments?. Mar. Ecol. Prog. Ser. 42: 33-38

Perez, K. T., Morrison, G. M., Lackie, N. F., Oviatt, C. A., Nixon, S. W., Buckley, B. A., Heltshe, J. F. (1977). The importance of physical and biotic scaling to the experimental simulation of a coastal marine ecosystem. Helgoländer wiss. Meeresunters. 30: 144-162

Peterson, W. T., Bellantoni, D. C. (1987). Relationships between water-column stratification phytoplankton cell size and copepod fecundity in Long Island Sound and off Central Chile. In: Payne, A. I. L., Gulland, J. A., Brink, K. H. (eds.) The Benguela and comparable ecosystems. S. Afr. J. mar. Sci. 5: 411-421

Rosenberg, G. G. (1980). Filmed observations of filter feeding in the marine planktonic copepod Acartia clausii. Limnol. Oceanogr. 25(4): 742-747

Rothschild, B. J., Osborn, T R. (1988). Small-scale turbulence and plankton contact rates. J. Plankton Res. 10: 465-474

Saiz, E., Alcaraz, M. (1991). Effects of small-scale turbulence on development time and growth of Acartia grani (Copepoda: Calanoida). J. Plankton Res. 13: 873-883

Strickler, J. R. (1985). Feeding currents in calanoid copepods: two new hypotheses. In: Laverack, M. S. (ed.) Physiological adaptations of marine animals. Symp. Soc. exp. Biol. 39: 459-485

Sundby, S., Fossum, P. (1990). Feeding conditions of ArctoNorwegian cod larvae compared with the RothschildOsborn theory on small-scale turbulence and plankton contact rates. J. Plankton Res. 12: 1153-1162

Tiselius, P., Jonsson, P. R. (1990). Foraging behaviour of six calanoid copepods: observations and hydrodynamic analysis. Mar. Ecol. Prog. Ser. 66: 23-33

Manuscript first received: November 14, 1991

Revised version accepted: February 4, 1992 